\title{
Sensitive and Label-Free Detection of miRNA-145 by Triplex Formation
}

\author{
Anna Aviñó ${ }^{1,3}$, César S. Huertas ${ }^{2,3}$, Laura M. Lechuga ${ }^{2,3}$ \& Ramon Eritja, ${ }^{1,3 *}$ \\ ${ }^{1}$ Dpt of Chemical and Biomolecular Nanotechnology. Institute for Advanced Chemistry \\ of Catalonia (IQAC), CSIC, Barcelona, Spain. \\ ${ }^{2}$ Catalan Institute of Nanoscience and Nanotechnology (ICN2), CSIC, ICN2 Building, \\ Campus UAB 08193 Bellaterra, Barcelona, Spain \\ ${ }^{3}$ CIBER-BBN Networking Center on Bioengineering, Biomaterials and Nanomedicine, \\ Barcelona, Spain. \\ e-mail: recgma@cid.csic.es, : 34-93-4006145; Fax: 34-93-2045904
}

This is a post-peer-review, pre-copyedit version of an article published in Analytical and Bioanalytical Chemistry. The final authenticated version is available online at https://doi.org/10.1007/s00216-015-9180-6 


\section{Abstract}

The development of new strategies for detecting miRNAs has become a crucial step in the diagnostic field. miRNA profiles depend greatly on the sample and the analytical platform employed, leading sometimes to contradictory results. In this work we study the use of modified parallel tail-clamps to detect a miRNA sequence involved in tumor suppression by triplex formation. Thermal denaturing curves and CD measurements have been performed to confirm that parallel clamps carrying 8-aminoguanine form the most stable triplex structures with their target miRNA. The modified tail-clamps have been tested as bioreceptors in a SPR biosensor for the detection of miRNA-145. The detection limit was improved 2.4 times demonstrating that a stable triplex structure is formed between target miRNA and 8-aminoguanine tail-clamp bioreceptor. This new approach is an essential step toward the label-free and reliable detection of miRNA signatures for diagnostic purposes.

Keywords Triplex, target miRNA-145, SPR biosensor, parallel tail-clamp, 8-amino-2'deoxyguanosine.

\section{Introduction}

MicroRNAs (miRNAs) are short RNA molecules (on average only 22 nucleotides long) that play important role in many basic biological processes including development, cell proliferation, differentiation and apoptosis [1]. Several thousands of miRNA have been identified as regulatory molecules of gene expression at the post-transcriptional level and also have been used as a new tool in medical diagnosis [2]. The mechanisms of action of miRNA are not yet fully characterized but expression levels of specific miRNA in tissues have already been correlated with several diseases. In fact detection of differential expression of miRNA can provide valuable diagnostic and prognostic data [3, 4].

Measuring miRNAs instead of traditional protein biomarkers is still a very challenging task. Given the miRNAs size and levels in cells, the detection methods need to be extremely sensible and selective in small amounts of complex RNA sample.

The Northern blot and real-time quantitative polymerase reaction (qPCR) are the most used methods for miRNA detection $[5,6]$. However, the first one requires large amounts of sample whereas the second one is not always accurate in miRNA measurements. In addition to these methods, microarray technology has been applied to parallelized detection of multiple miRNAs [7]. In this case it is particularly difficult to design very specific probes for a number of miRNAs and sometimes also to distinguish real signals from non specific hybridization. Differences in microRNA detection levels are technology and sequence dependent [8]. Several reviews have been published dealing with microRNA detection and quantification by conventional amplification-based molecular strategies or by using amplification-free biosensor-based methods [9-11]. These last techniques have been used to complement the first ones although recent results have shown that biosensors can deliver high sensitivity (aM-nM levels), minimal sample preparation and 
rapid measurements that provide excellent characteristics for clinical diagnosis as well as fundamental research. The main molecular recognition event in miRNA biosensors is the hybridization of target miRNA with a typically DNA probe of complementary sequence. The quantitative recognition is transduced in a measurable electrochemical [12], electromechanical [13] or optical signal [14]. Surface plasmon resonance (SPR) offered rapid, sensitive and on-site analysis of miRNA [14]. This method has been applied to quantify mature mir-122 in a complex sample at subfemtomole level [14]. The detection protocol is based on the recognition of DNA-RNA hybrid duplex by a specific antibody. In addition, a method for detection of miRNA with excellent sensitivity has been described by SPR imaging and DNAcoated nanoparticles [15]. All the described methods rely on duplex formats between the miRNA and the complementary probe. Nevertheless, alternative formats can also be used to detect miRNA. Triple-helix formation based on Hoogsteen base pairing to the Watson-Crick duplex is an interesting alternative. Triplex can only be formed at specific polypurine-polypyrimidine sequences but these triple-helix target sites are widespread within the human genome, especially at promoter regions [16-18].

Previously, our group reported the triplex-stabilizing properties of 8-aminopurines [19, 20] and the use of parallel and antiparallel tail clamps to increase the efficiency of triplex formation with structured DNA and RNA targets [21]. All this knowledge was applied to develop a new affinity capture technique based on the stability of triplex by using tail-clamps carrying 8-aminopurines as bioreceptor [22)]. Recently, we applied this triplex affinity capture strategy for the detection of Listeria mRNA using a SPR biosensor [23].

Here, we have described the triple-helix formation of parallel clamps containing 8-amino-2'deoxyguanosine and miRNA-145. This miRNA has been shown to act as a tumor suppressor, as in most of the human cancer cell lines, a clear reduction of the expression of this miRNA was observed. In particular, low-expression in human colon or gastric cancer cells has been reported [24], with a clear relationship between the expression of miRNA-145 and the clinicopathological findings in human colorectal tumors. On the basis of such findings, we have developed a new strategy to capture and measure miRNA-145 by SPR based in the formation of stable triplex structures.

\section{Materials and Methods}

\section{Synthesis of Oligonucleotides}

Oligonucleotides used in this study are listed in Table $1 \mathrm{~S}$ and $2 \mathrm{~S}$. The parallel-stranded clamps were designed to carry the polypurine sequence with additional bases complementary to miRNA-145, connected head-to-head and through their 3'-ends with the Hoogsteen C, T sequence. A tetrathymidine sequence was used in the loop to connect the two strands. First, the purine part was assembled by using standard phosphoramidites for the natural bases and 8-aminoguanine phosphoramidite from commercial sources, then a tetrathymidine loop sequence was added. Finally, the heptapyrimidine sequence was prepared by using reversed $\mathrm{C}$ and $\mathrm{T}$ phosphoramidites. 
Oligonucleotides for biosensing were prepared as described with additional 15 thymidines and 5 , thiol modifier-C6 S-S CE phosphoramidite (Link Technologies) in the 5'-end.

Sequences were prepared on an Applied Biosystems 3400 synthesizer using polystyrene solid supports (LV200) according to the protocols of the manufacturer. The dimethoxytrityl group of the last phosphoramidite was not removed to facilitate reversed-phase purification (DMT-on protocol). Standard protecting groups were used for DNA sequences $\left(A^{B z}, G^{i b u}, C^{B z}, T\right)$. The RNA oligonucleotide, miRNA145, was synthesized using 2'-O-TBDMS protected phosphoramidites ( $\mathrm{A}^{\mathrm{Bz}}, \mathrm{G}^{\mathrm{dmf}}, \mathrm{C}^{\mathrm{Ac}}$ and $\left.\mathrm{U}\right)$. Oligonucleotides containing 8-AminoG were prepared using commercial available phosphoramidite (Berry \& Associates). After assembling of the sequences, oligonucleotide supports were treated with aqueous ammonia $(32 \%)$ for $16 \mathrm{~h}$ at $55^{\circ} \mathrm{C}$ for DNA sequences and $1 \mathrm{~h}$ at $55^{\circ} \mathrm{C}$ for RNA sequence. Addition of $\beta$-mercaptoethanol was used in the deprotecting solutions in sequences containing 8 -AG. The ammonia solutions were concentrated to dryness and for DNA sequences the product was desalted on NAP-10 (Sephadex G-25) columns eluted with water. For miRNA-145, after ammonia treatment, the residue was dissolved in a total volume of $85 \mu \mathrm{L}$ of $1 \mathrm{M}$ TBAF in THF and rocked at room temperature for $12 \mathrm{~h}$. Then, $85 \mu \mathrm{L}$ of $1 \mathrm{M}$ triethylammonium acetate (TEAA) and $330 \mu \mathrm{L}$ of water were added to the solution. The oligonucleotide was desalted in a NAP-5 column using water as the eluent and evaporated to dryness. All the oligonucleotides were purified by HPLC (DMT-ON). Oligonucleotides for biosensing were deprotected with aqueous ammonia $(32 \%)$ for $16 \mathrm{~h}$ at $55^{\circ} \mathrm{C}$ with $0.1 \mathrm{M}$ DTT.

Column: Nucleosil 120-10 $\mathrm{C}_{18}(250 \times 4 \mathrm{~mm}) ; 20 \mathrm{~min}$ linear gradient from $15 \%$ to $80 \% \mathrm{~B}$ and 5 $\min 80 \% \mathrm{~B}$, flow rate $3 \mathrm{~mL} / \mathrm{min}$; solution $\mathrm{A}$ was $5 \% \mathrm{ACN}$ in $0.1 \mathrm{M}$ aqueous TEAA and $\mathrm{B} 70 \% \mathrm{ACN}$ in $0.1 \mathrm{M}$ aqueous TEAA. The pure fractions were combined and evaporated to dryness. The residue that was obtained was treated with $1 \mathrm{~mL}$ of $80 \% \mathrm{AcOH}$ solution and incubated at room temperature for $30 \mathrm{~min}$. The aqueous phase is extracted with diethylether and, finally, the deprotected oligonucleotide was desalted in a NAP-10 column. All oligonucleotides were quantified by absorption at $260 \mathrm{~nm}$ and confirmed by MALDI mass spectrometry (see supplementary Table S1 and S2 online).

\section{Thermal UV Denaturation and CD studies}

The absorbance versus temperature curves of duplexes and triplexes were measured at $1.7 \mu \mathrm{M}$ strand concentration in $0.1 \mathrm{M}$ sodium phosphate/citric acid buffer at $\mathrm{pH} 5$ and $\mathrm{pH} 6$ or in $0.1 \mathrm{M}$ sodium phosphate buffer at $\mathrm{pH} 7$, all the buffers are implemented with $1 \mathrm{M} \mathrm{NaCl}$. Thermal UV experiments were performed in Teflon-stopped $1 \mathrm{~cm}$ path length quartz cells on a JACSO V-650 spectrophotometer equipped with thermoprogrammer. The samples were heated to $90^{\circ} \mathrm{C}$, allowed to slowly cool down to $20^{\circ} \mathrm{C}$, and then warmed during the denaturation experiments at a rate of $0.5^{\circ} \mathrm{C} / \mathrm{min}$ to $85^{\circ} \mathrm{C}$, monitoring absorbance at $260 \mathrm{~nm}$ and $300 \mathrm{~nm}$. The data were analyzed by the denaturation curve processing program, MeltWin v. 3.0. Melting temperatures $\left(\mathrm{T}_{\mathrm{m}}\right)$ were determined by computerfit of the first derivative of absorbance with respect to $1 / \mathrm{T}$.

$\mathrm{CD}$ measurements were recorded at the same strand concentration as denaturing curves. Spectra were recorded on a JASCO spectropolarimeter J-810 and registered at $15^{\circ} \mathrm{C}$ over a range of $205-320 \mathrm{~nm}$ 
with a scanning speed of $100 \mathrm{~nm} / \mathrm{min}$, a response time of $4 \mathrm{~s}, 0.5 \mathrm{~nm}$ data pitch and $1 \mathrm{~nm}$ bandwidth. The samples were dissolved in the above buffers annealed and slowly cooled down to room temperature and left at $4^{\circ} \mathrm{C}$ at least one night.

\section{Experimental Procedure for Detection Using the SPR Biosensor}

SPR biosensor device: We employed a home-made $\mathrm{SPR}^{23}$. The SPR sensor platform is based on Kretschmann configuration, monitoring the binding events in real time. A p-polarized light of $670 \mathrm{~nm}$ from a laser source is divided in two identical beams focused on the crystal-backside of the sensor chip (glass surface coated with $2 \mathrm{~nm}$ of chromium and $45 \mathrm{~nm}$ of gold, 10x10x0.3 mm). Measurements are performed at a fixed angle of incidence. Variations of the refractive index (RI) due to the biointeraction events occurring at the sensing surface are detected as changes in the reflected light intensity by a multielement photodiode, permitting a label-free detection. The flow system consists of two flow cells (300 $\mathrm{nL}$ each) for two simultaneous and independent analysis. The device incorporates all optics, electronics and fluidics components necessary to operate autonomously. Sensograms reproduce the binding events by monitoring the increase (or decrease in case of unbinding events) of the intensity of the reflected light ( $\Delta$ Reflectivity (\%), $\Delta \mathrm{R}(\%))$ vs. time (seconds, s). This change of the intensity of the reflected light is directly related to changes in the RI of the dielectric medium caused by mass changes (as a biomolecular interaction) on the metal surface.

Gold sensor chips (SSens, Enschede -The Netherlands-) are used as the sensing element. They are cleaned prior to experiments by rinsing with solvents of decreasing polarity (i.e. trichloroethylene, acetone, ethanol and $\mathrm{dH}_{2} \mathrm{O}$ ) previously heated up to their boiling point. Then, sensor chips are dried under nitrogen $\left(\mathrm{N}_{2}\right)$ flux and dipped into piranha solution $\left(\mathrm{H}_{2} \mathrm{SO}_{4}: \mathrm{H}_{2} \mathrm{O}_{2}\right.$ volume ratio 7:3) for a few seconds, rinsed again with water and dried under $\mathrm{N}_{2}$ flux.

Bioreceptor immobilization: The different bioreceptors were tested in independent assays. Clean gold biosensor chips were coated ex situ by $100 \mathrm{~mL}$ of immobilization solutions $(1 \mu \mathrm{M}$ DNA receptor with 50 nM Mercaptohexanol (MCH) (Sigma Aldrich, Steinhem -Germany-) in PB buffered solution (50 mM Phosphate buffer- $0.75 \mathrm{mM} \mathrm{NaCl}, \mathrm{pH} 7)$ ) and kept in a humid chamber for $3 \mathrm{~h}$. Then, they were rinsed in DEPC treated $\mathrm{H}_{2} \mathrm{O}$, dried under $\mathrm{N} 2$ flux and placed in the sensor device.

Detection was done in situ at room temperature (RT) by flowing different concentrations of miRNA-145 over the sensor surface for their hybridization with the different immobilized bioreceptors at a $16 \mu \mathrm{L} / \mathrm{min}$ constant rate. These samples were dissolved in $5 \mathrm{X}$-SSC solution $(0.75 \mathrm{M}$ in $\mathrm{NaCl}, 0.075 \mathrm{M}$ in sodium citrate, $\mathrm{pH} 7$ ). Regeneration after hybridization was achieved by injecting a $35 \%$ formamide solution, allowing the analysis of a high number of interactions using the same bioreceptor monolayer.

Material sterilization: To keep RNase free conditions during measurements, sensor microfluidic was cleaned by flowing sequentially SDS $0.5 \%, \mathrm{HCl} 0.1 \mathrm{M}$, EtOH $100 \%$, NaOH $0.1 \mathrm{M}$ and sterile $\mathrm{H}_{2} \mathrm{O}$. The buffers and other solutions were prepared by using $\mathrm{H}_{2} \mathrm{O}$ milliQ incubated $\mathrm{O} / \mathrm{N}$ with $2 \%$ DEPC and autoclaved at $121^{\circ} \mathrm{C}$ during $30 \mathrm{~min}$. All solid materials were autoclaved at $121^{\circ} \mathrm{C} / 20 \mathrm{~min}$ for plastic materials and $134^{\circ} \mathrm{C} / 10 \mathrm{~min}$ for glass ones. 
Data analysis: The data were collected using Origin 8.0 software (OriginLab, Northampton, MA). The experimental detection limit was defined as the target concentration giving a $\Delta \mathrm{R}(\%)$ in the hybridization signal at least three times higher than that of the standard deviation of RNA control signal. Limits of quantification were defined as the target concentration giving $\Delta \mathrm{R}(\%)$ in the hybridization signal at least ten times higher than that of the standard deviation of the RNA control signal.

\section{Results}

We designed and synthesized parallel clamps (PT Clamp, 3AG PT Clamp, Fig. 1a) targeting a 7homopyrimidine sequence of miRNA-145 in order to assess their capacity to form triple helices. The homopurine portion is extended in order to hybridize the target miRNA-145 with Watson-Crick interactions and the inverted homopyrimidine portion of the clamps should form a triplex by Hoogsteen hydrogen bonding. Base-pairing scheme of the triads that forms the triplex structures is showed in Figure 1b. 3AG PT Clamp contains three consecutive 8 -aminoguanines (8-aminoGs). The 7-base polypyrimidine track of the miRNA-145 is shorter than 12-bases used in previous studies [23]. To overcome this problem and to improve the triplex formation we decided to use 8 -aminoG modification instead of 8 -aminoA due to their improved triplex formation efficacy. 8-aminoG increased triplex stability around $10{ }^{\circ} \mathrm{C} /$ per substitution in contrast to $7{ }^{\circ} \mathrm{C}$ of 8 -aminoA [19]. Complementary sequences to miRNA-145 were prepared with or without 8-aminoG to study the effect of this derivative in RNA-DNA Watson-Crick duplexes. Formation of stable triplex structures was confirmed by UV denaturing curves and CD measurements. 
b
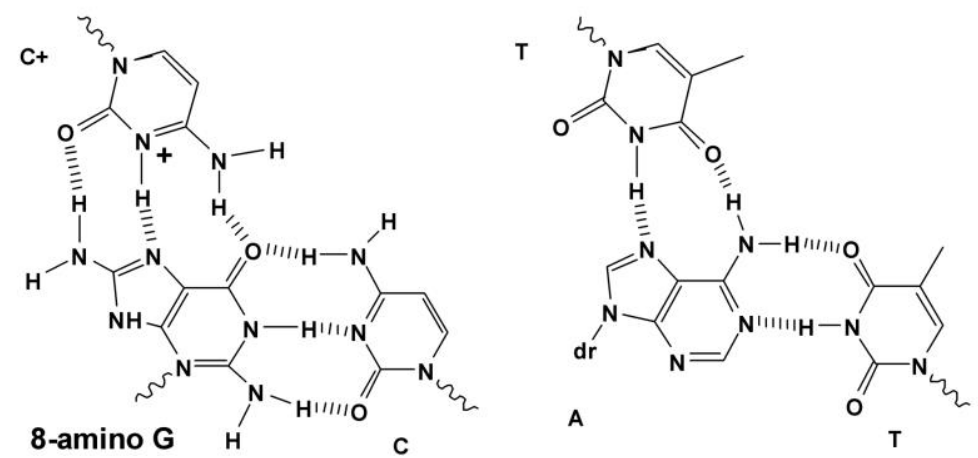

Fig.1 Scheme showing the binding of tail-clamps and complementary sequences to miRNA-145 yielding triplex and duplex structures respectively (a) and scheme showing the triads that forms triplex structures $\mathrm{C}^{+}$-8-aminoG - C and T-A-T (b).

\section{Thermal UV and CD Studies of Duplex and Triplex Structures with miRNA-145}

The thermal stability of the complex formed between parallel clamps and miRNA-145 was analyzed at pH 5 which should be optimal for parallel-triplex formation (Table 1). A single transition was observed at $260 \mathrm{~nm}$ that corresponds to the denaturation of either triplex or duplex to single stranded (SS) form (Fig. 2a). The presence of triplex was confirmed by measuring the melting curves at $300 \mathrm{~nm}$. At this wavelength an increased on the UV-absorption is observed (Fig. 2b) only if triplex is formed as the transition is due to the deprotonation of cytosine [21,22]. Moreover, 3AG PT clamp forms a more stable triplex than with PT clamp. Also it was observed that the melting temperature of the transition followed at $300 \mathrm{~nm}$ is lower than the transition followed by $260 \mathrm{~nm}$, indicating that the Hoogsteen polypyrimidine strand dissociates before the strands forming the Watson-Crick duplex. However, at higher $\mathrm{pH}$ values (pH 6-7), only 3AG PT clamps are capable of forming stable triplexes, and the melting temperature is similar from the measurement performed at $260 \mathrm{~nm}$ and at $300 \mathrm{~nm}$. Single transitions were also observed at $\mathrm{pH} 7$ but in this case the transition became broad. 


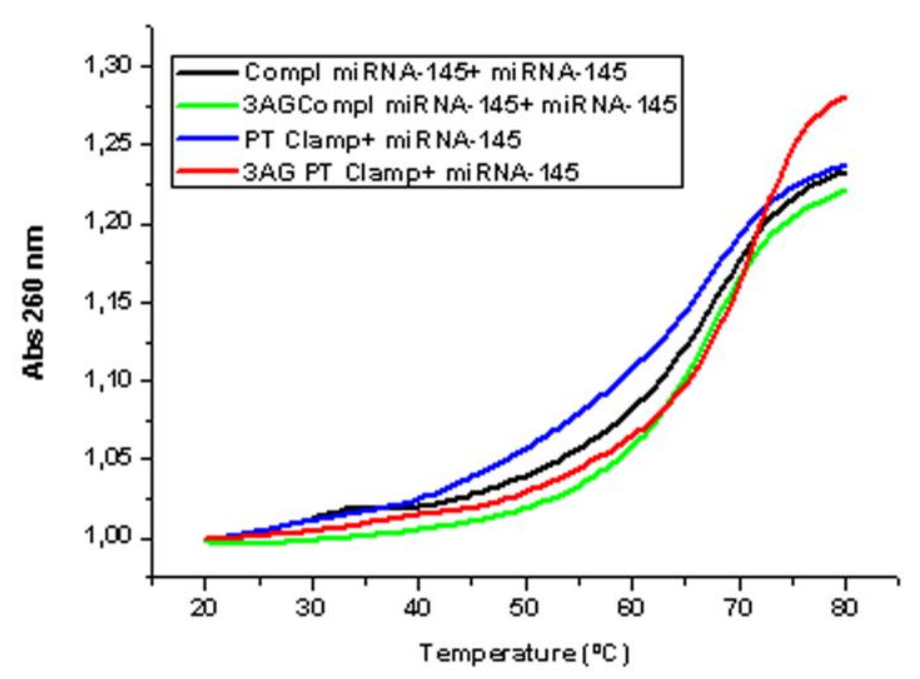

b

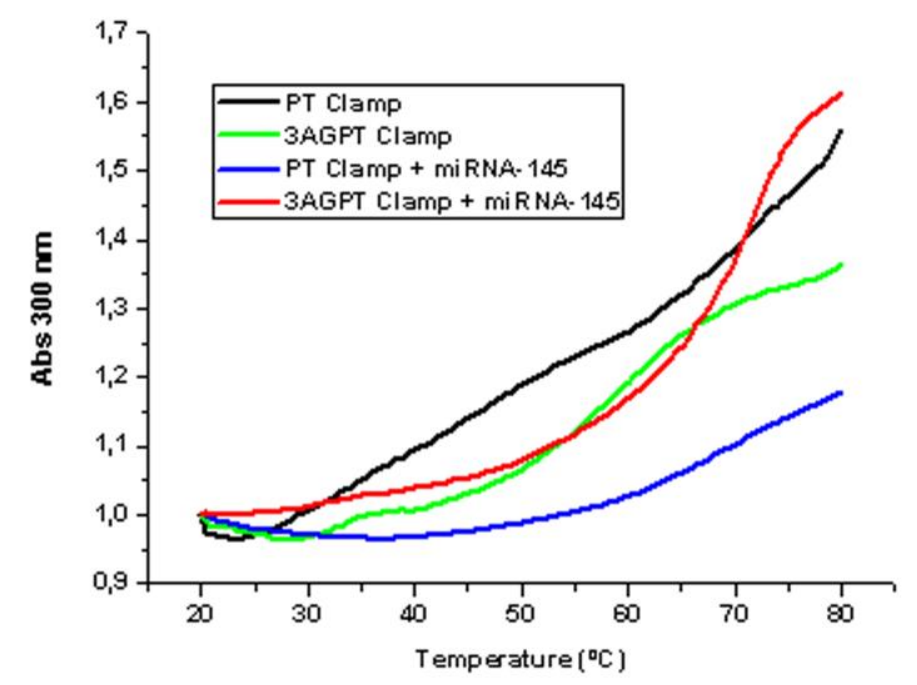

Fig. 2 UV Melting Curves. (a) Normalized UV melting curves of complementary mi-RNA145 sequences and PT Clamps with miRNA-145 at $260 \mathrm{~nm}$. (b) Normalized UV melting curves of PT Clamps alone and with miRNA-145 at $300 \mathrm{~nm}$.

Additionally, we also measured the transition between control clamps in the presence or in the absence of 8-aminoG in which the pyrimidine strand was not appropriate to form triplex structure. In this case, no triplex transition was observed and the results were similar to duplex transition (Table 1).

Table 1. Melting Temperatures (Tm) of Duplexes and Triplexes. Conditions: $0.1 \mathrm{M}$ sodium phosphate buffer and citric acid at the appropriate $\mathrm{pH}$ with $1 \mathrm{M} \mathrm{NaCl}$. The first value corresponds to the value of the Tm obtained measuring the absorption at $260 \mathrm{~nm}$ and the second one correspond to the value obtained if the melting curve is measured at $300 \mathrm{~nm}$. Uncertainties in Tm values are estimated at $\pm 1{ }^{\circ} \mathrm{C}$ 


\begin{tabular}{|c|l|l|l|l|}
\hline Sequences & $\begin{array}{l}\text { Expected } \\
\text { transition }\end{array}$ & Tm $\left({ }^{\circ} \mathrm{C}\right) \mathrm{pH} 5$ & $\mathrm{Tm}\left({ }^{\circ} \mathrm{C}\right) \mathrm{pH} 6$ & $\mathrm{Tm}\left({ }^{\circ} \mathrm{C}\right) \mathrm{pH} 7$ \\
\hline $\begin{array}{c}\text { PT Clamp + miRNA- } \\
145\end{array}$ & Triplex $\rightarrow \mathrm{SS}$ & $63.7 / 56.8$ & $65.0 /$ none & $66.0 /$ none \\
\hline $\begin{array}{c}\text { 3AG Clamp + } \\
\text { miRNA-145 }\end{array}$ & Triplex $\rightarrow \mathrm{SS}$ & $75.9 / 68.7$ & $71.4 / 71.0$ & $57.5 / 58$ \\
\hline PT Clamp & $\begin{array}{l}\text { Parallel } \\
\text { duplex } \rightarrow \mathrm{SS}\end{array}$ & 50.5 & 49.8 & 48.2 \\
\hline 3AG Clamp & $\begin{array}{l}\text { Parallel } \\
\text { duplex } \rightarrow \mathrm{SS}\end{array}$ & 63.5 & 58.6 & 39.4 \\
\hline $\begin{array}{c}\text { Compl miRNA-145 + } \\
\text { miRNA-145 }\end{array}$ & $\begin{array}{l}\text { Antiparallel } \\
\text { duplex } \rightarrow \mathrm{SS}\end{array}$ & 64.1 & 67.2 & 67.7 \\
\hline $\begin{array}{c}\text { 3AGCompl miRNA- } \\
\text { 145 + miRNA-145 }\end{array}$ & $\begin{array}{l}\text { Antiparallel } \\
\text { duplex } \rightarrow \mathrm{SS}\end{array}$ & 68.9 & 57.5 & 57.8 \\
\hline $\begin{array}{c}\text { Control Clamp+ } \\
\text { miRNA 145 }\end{array}$ & $\begin{array}{l}\text { Antiparallel } \\
\text { duplex } \rightarrow \mathrm{SS}\end{array}$ & 68.5 & 57.0 & 66.9 \\
\hline $\begin{array}{c}\text { 3AG Control } \\
\text { Clamp+miRNA-145 }\end{array}$ & $\begin{array}{l}\text { Antiparallel } \\
\text { duplex } \rightarrow \mathrm{SS}\end{array}$ & 64.6 & 64.9 & 65.9 \\
\hline
\end{tabular}

Thermal stability of parallel clamps alone (without miRNA target) was also studied at different pHs (Table 1). Thermal UV melting techniques of unmodified PT Clamp showed almost similar Tm in all pHs in contrast to the 3AG Clamp, that showed a strong dependence with the pH. The stability of 3AG Clamp is decreased with the $\mathrm{pH}$. At $\mathrm{pH} 5$, the difference between $3 \mathrm{AG}$ and the unmodified clamp is $13^{\circ} \mathrm{C}$ whereas at pH 7 the unmodified clamp has a Tm higher than the 3AG Clamp does. Hoosgsteen hydrogen bonds are affected by the protonation of the cytosines, so that at a lower $\mathrm{pH}$ they are more stable. This is the case of 3 AG PT clamp. By contrast, the PT clamp is almost not affected by the $\mathrm{pH}$. In this case, the main and most stable species may be a bimolecular antiparallel Watson-Crick duplex formed by the purine track of one clamp and the pyrimidine track of a second molecule in opposite direction. The parallel clamp may be a minor component. This is also in agreement with the similar melting temperature of the 3 AG clamp at $300 \mathrm{~nm}$ and at $260 \mathrm{~nm}$ at pH 5 and 6 . Absorbance changes at a longer wavelength have been described to indicate transitions in which the protonation of cytosines are required [25].

Melting temperatures of heteroduplex formed by mixing miRNA-145 and the DNA complementary strand were similar at pH 6 and 7 but the presence of 8 -aminoG decreased the stability of these duplex. In contrast to this, at pH 5 the duplex with 8-aminoG is more stable than the unmodified duplex. Secondary structures of mi-RNA145, Compl miRNA-145 and 3AG compl miRNA-145 were discarded as no melting was observed at any studied pHs.

The CD spectra of the triplex formed between the clamps and the target miRNA-145 and as well as the clamps alone were analyzed at pH 6 and are shown in Figure 3. The appearance of a more intense negative band near 210-220 $\mathrm{nm}$ in the CD spectra indicates the formation of a triplex [26-28]. 

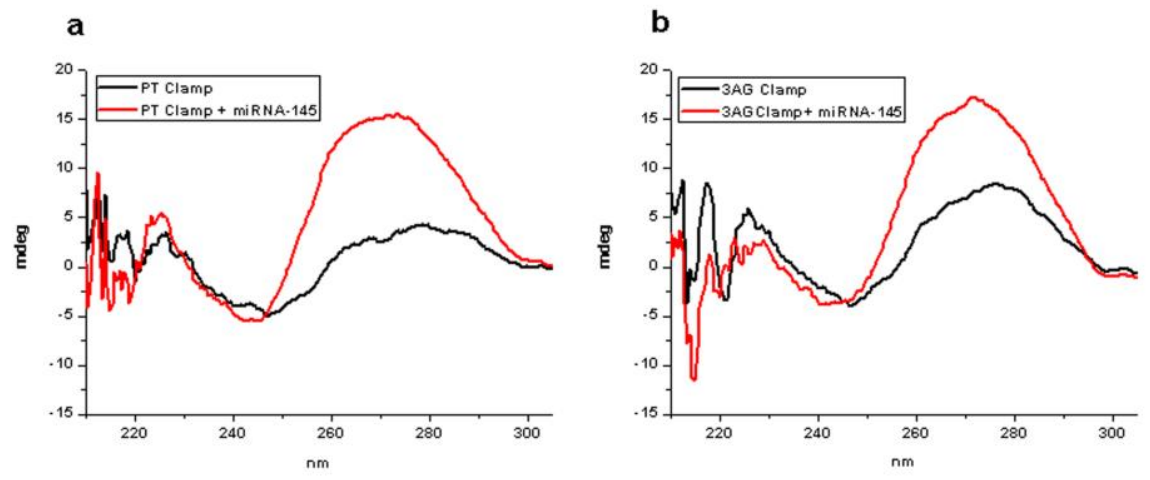

Fig. 3 CD spectra of PT Clamp alone and with miRNA-145 (a) and 3AG PT Clamp alone and with miRNA-145 (b) in $100 \mathrm{mM}$ phosphate buffer and $1 \mathrm{M} \mathrm{NaCl} \mathrm{pH} 6$.

\section{Detection of miRNA-145 Using Parallel Clamps}

SPR biosensor offers rapid, sensitive and label-free analysis of nucleic acids sequences. This technique uses complementary DNA bioreceptors immobilized in a gold sensor chip to target specific nucleic acid sequences. The binding event is detected by alterations of the optical properties of the gold surface. Minute variations in the refractive index caused by the nucleic acid hybridization change the intensity of the reflected light, permitting the real time monitoring of the capture. In this work, we analyzed the use of tail clamps bioreceptors immobilized on the gold sensor chip for the detection of miRNA-145 (Fig. 4a). Previously reported results with SPR biosensor using tail clamps carrying 8aminoadenine as bioreceptors showed that, this approach was able to successfully detect structured and long RNAs such as mRNA of Listeria [23]. The pyrimidine track of the tail clamps contained 12 nucleotides providing quite stable triplex structures. However, for the detection of miRNA we face an important challenge since miRNAs are small RNA molecules and the Hoogsteen polypyrimidine tracks are shorter (7 nucleotides for the miRNA-145) which might affect the stability of the clamp and the hybridization efficiency to form triplexes. For this reason, we used 8-aminoG instead of 8-aminoA to improve the triplex stability. 
a

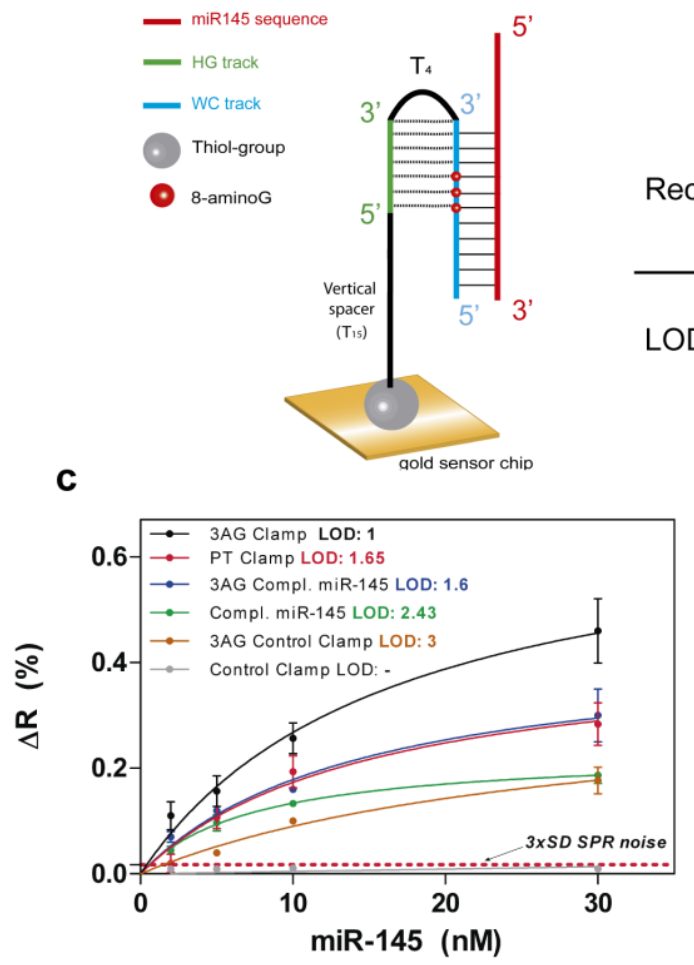

b

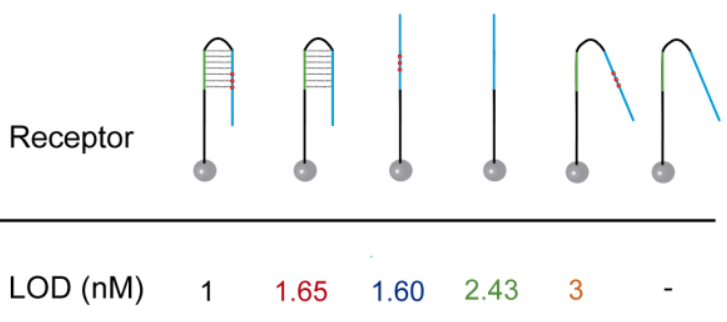

d

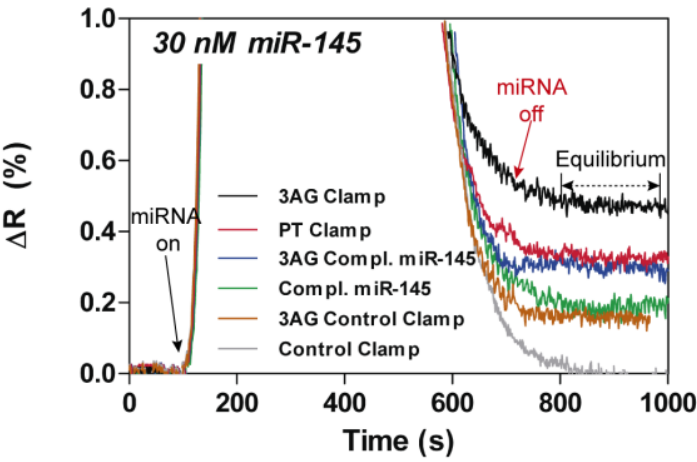

Fig. 4 Scheme of the gold sensor chip functionalized with the 3AG PT Clamp bioreceptor (a). Schemes of the different bioreceptors employed for the SPR biosensor study. LODs obtained for each bioreceptor are indicated (b). SPR calibration curves of the detection of miR-145 with different bioreceptors. The sensitivity for each bioreceptor was considered as three times the baseline noise standard deviation (discontinuous red line) All data show mean \pm SD from the SPR-equilibrium signal of triplicate measurements (c). Real-time sensograms of $30 \mathrm{nM}$ miRNA-145 using the different bioreceptor variants Dashed arrow indicates the equilibrium signal of the SPR sensograms (d).

To assess the advantage of this new triplex structure as a bioreceptor in a SPR biosensor for the detection of miRNAs, we designed two PT clamp bioreceptors, one modified 8-aminoG (3AG Clamp) and other with no 8-aminoG modification (PT Clamp). For comparison purposes, we also assayed duplex formation capture in which complementary sequences to miRNA-145 were prepared with and without 8aminoG (3AG Compl. miR-145 and Compl. miR-145, respectively). In addition, we analyzed tail clamp controls which include incorrect polypyrimidine sequence in which the triplex was unable to form properly (3AG Control Clamp and Control Clamp). Schematic representations of all the employed bioreceptors are depicted in Figure 4b. Bioreceptor sequences are shown in Table 2S.

For the gold sensor chip functionalization, all bioreceptors were modified with a thiol-group at the 5'-end of the Hoogsteen pyrimidine track for their attachment to the gold surface by chemisorption through thiol-gold chemistry. We include a block of 15 thymidine nucleotides (Poly- $\left.\mathrm{T}_{15}\right)$ as a vertical spacer in order to improve the accessibility of the miR-145 target to the bioreceptor monolayer as previously reported ${ }^{23}$. In addition, we used 6-mercapto-1-hexanol as a lateral spacer to minimize steric hindrance ${ }^{29}$. Gold sensor chips were coated ex situ with the immobilization solutions and were kept under 
an humid chamber for $3 \mathrm{~h}$. During the incubation time, thiolated bioreceptors arrange themselves spontaneously into a so-called self-assembled monolayer (SAM).

Results of SPR detection of miRNA-145 with all the bioreceptors are displayed in Figure 4 b-d. We evaluated the efficiency of all the bioreceptors by monitoring their response to standard solutions at different miR-145 concentrations ranged from $2 \mathrm{nM}$ to $30 \mathrm{nM}$. Triplicate measurements of each miR-145 concentration were obtained and calibration curves for each bioreceptor have been represented (Figure 4c and Figure S1 Electronic Supplementary Material). The limit of detection (LOD) of each bioreceptor has been calculated and listed in Figure 4b. The specificity of the bioreceptor was confirmed by measuring the sensogram with a different miRNA (miRNA-194) with similar GC content (Figure S2, Electronic Supplementary Material).

Real-time sensograms of miR-145 at $30 \mathrm{nM}$ with each bioreceptor (Figure 4d) showed an increase in the sensor response of miRNA-145 for the PT clamp bioreceptors compared with the duplex ones. In addition, their sensitivities were improved exhibiting more than 1.5 times lower LODs. On the other hand, 8-aminoG modifications seem to largely stabilize the hybridization, obtaining higher signals with bioreceptors containing them as compared with the non-modified ones. The duplex detection approach is favored when the complementary bioreceptor is modified with 8-aminoG in accordance with the UVdenaturing data. Furthermore, the introduction of these modifications in the PT Clamp bioreceptor promoted the stabilization of the triplex as they showed the maximum biosensor response, leading to an enhancement of the sensitivity. A LOD of $1 \mathrm{nM}$ was determined for 3AG Clamp bioreceptor, rendering in a LOD 2.43 times lower as compared to the standard duplex bioreceptor. Surfaces biofunctionalized with unmodified PT Clamp receptors generated a LOD of $1.65 \mathrm{nM}$, very similar to the one obtained for the duplex-forming 3AG Compl. bioreceptor $(1.60 \mathrm{nM})$. This result suggests that under our experimental conditions, a triplex structure is only formed with 8-aminoG-modified parallel tail-clamps.

\section{Discussion}

Searching new strategies for detecting miRNA has become an intensive research field. miRNA profiles depend greatly on the sample and on the analytical platform and protocols used and results are not always consistent. Developing a different sensitive and label-free method will have a potential application in profiling miRNA expression as signatures for clinical diagnostics.

U.V denaturing and CD measurements have been used to study the formation of stable triplex structures with tail-clamps and miRNA-145. The introduction of 8-amino-2'-deoxyguanosine derivatives into parallel tail clamps are the key in the formation of stable triplex structures when the binding pyrimidine sequence of the target miRNA-145 is relatively short. These results were applied to provide a new and sensitive approach for label-free detection of miRNA-145 using a SPR biosensor. A stable triplex structure was formed at a neutral $\mathrm{pH}$ by using parallel tail-clamps bioreceptors modified with 8aminoguanine derivatives with a short polypyrimidine track. 
This modified tail-clamp was more efficient for target detection than the conventional duplex formats. The introduction of 8 -aminoG in both triplex- and duplex-capture probes was found to stabilize the hybridization, improving the LODs in more than 1.5 times. The sensitivity of this approach provided levels that are in the range of the reported optical miRNA biosensors [11].

This novel approach is not universal but offers great potential for miRNA detection or in any other RNA structure; in this case the requirement was to have only seven pyrimidines in the sequence in order to design an appropriate tail-clamp receptor. Sequence analysis over one hundred of reported human miRNA was conducted. More than one third of miRNA sequences contained tracks of up to seven pyrimidines in their sequence. In addition, modified PT clamps may be redesigned to cope with small interruptions in the polypyrimidine target sequence [19] and accordingly, more miRNA sequences could be detected using this approach.

The specificity of this method comes from the extended purine track of the PT clamps that recognize the miRNA sequence. The purine track is extended with several nucleotides being perfectly complementary to miRNA though Watson and Crick hydrogen bonds. The addition of these nucleotides stabilizes the clamp and increases the specificity of the recognition for their target.

Stable triplex have been described between double helix DNA and miRNA. There is evidence that transcriptional gene silencing over promoter regions mediated by triplex structures occurs in the cell [30, 31] and the complex miRNA-DNA.DNA triplex has been found that is stabilized by proteins [32]. In particular, Triplex-forming complexes between human miRNAs (hsa-miR) that are complementary to human immunodeficiency virus (HIV-1) have been explored as antiviral therapeutic agents [33]. All these studies show the interest on triplex formation in the field of miRNA and other RNA regulatory elements. This work will expand this interest towards the detection of therapeutic miRNA that can form stable triplexes. These specific polypurine-polypyrimidine sequences are abundant at promoter regions [16-18]

More work is still needed to extend this study to the detection of miRNA containing pyrimidine tracks with small number of polypyrimidine tracks or to miRNA having interruptions in the polypurinepolypyrimidine track. The achieved results are an exceptional advance toward the detection of miRNA for diagnostic and biomedical purposes. As the detection of the differential expression of microRNA is important in the prognostic and prediction of several diseases, we believe that these findings are significant in producing a new approach to detect miRNA and in the development of a general sensing platform for miRNA detection in biological samples.

\section{Acknowledgments}

This work was partially supported by grants of the Spanish Ministry of Economy (MINECO) (CTQ201020541 and CTQ2014-52588-R), Generalitat de Catalunya (2009/SGR/208, 2014/SGR/624) and CIBERBBN. CIBER-BBN is an initiative funded by the VI National R\&D\&i Plan 2008-2011, Iniciativa Ingenio 2010, Consolider Program, CIBER Actions and financed by the Instituto de Salud Carlos III with assistance from the European Regional Development Fund. 
Conflict of Interest: The authors declare that they have no conflict of interest

\section{References}

1. He L, Hannon GJ (2004) MicroRNAs: small RNAs with a big role in gene regulation. Nature Reviews Genetics 5: 522-531.

2. Debernardi S, Skoulakis S, Molloy G, Chaplin T, Dixon-McIver A, Young BD (2007) MicroRNA miR-181a correlates with morphological sub-class of acute myeloid leukaemia and the expression of its target genes in global genome-wide analysis, Leukemia 21: 912-916.

3. He H, Jazdzewski K, Li W, Liyanarachchi S, Nagy R, Volinia S, Calin GA, Liu C, Franssila K, Suster S, Kloos RT, Croce CM, de la Chapelle A (2005) The role of microRNA genes in papillary thyroid carcinoma. Proceedings of the National Academy of Sciences USA 102:19075-19080.

4. Ikeda S, Kong SW, Lu J, Bisping E, Zhang H, Allen PD, Golub TR, Pieske B, Pu WT (2007). Altered microRNA expression in human heart disease, Physiological Genomics 31: 367-373

5. Lagos-Quintana M, Rauhut R, Yalcin A, Meyer J, Lendeckel W, Tuschl T. (2002) Identification of tissue-specific microRNAs from mouse. Current Biology, 12: 735-739

6. Benes V, Castoldi M (2010) Expression profiling of microRNA using real-time quantitative PCR, how to use it and what is available. Methods, 50: 244-249

7. Thomson JM, Parker J, Perou CM, Hammond SM (2004) A custom microarray platform for analysis of microRNA gene expression, Nature Methods, 1: 47-53

8. Leshkowitz D, Horn-Saban S, Parmet Y, Feldmesser E (2013) Differences in microRNA detection levels are nanotechnology and sequence dependent. RNA, 19: 527-538

9. Degliangeli F, Pompa PP, Fiammengo R (2014) Nanotechnology-based strategies for the detection and quantification of microRNA, Chemistry an European Journal 20: 9476-9492 
10. Dong H, Lei J, Ding L, Wen Y, Ju H, Zhang X (2013) MicroRNA: function, detection, and bioanalysis. Chemical Reviews 113: 6207-6233

11. Johnson BN, Mutharasan R (2014) Biosensor-based microRNA detection: techniques, design, performance, and challenges. Analyst 139: 1576-1588

12. Dong H, Jin S, Ju H, Hao K, Xu LP, Lu H, Zhang X (2012) Trace and label-free microRNA detection using oligonucleotide encapsulated silver nanoclusters as probes. Analytical Chemistry 84: 8670-8674

13. Johnson BN, Mutharasan R (2012) Sample preparation-free, real-time detection of microRNA in human serum using piezoelectric cantilever biosensors at attomole level. Analytical Chemistry 84: 1042610436

14. Sípová H, Zhang S, Dudley AM, Galas D, Wang K, Homola J, (2010) Surface plasmon resonance biosensor for rapid label-free detection of microribonucleic Acid at bubfemtomole level, Analytical Chemistry 82: 10110-10115

15. Fang S, Lee HJ, Wark AW, Corn RM (2006) Attomole microarray detection of microRNAs by nanoparticle-amplified SPR Imaging measurements of surface polyadenylation reactions. Journal of the American Chemical Society 128: 14044-14046

16. Morgan AR, Wells RD (1968) Specificity of the three-stranded complex formation between doublestranded DNA and single-stranded RNA containing repeating nucleotide sequences. Journal of Molecular Biology 37: 63-80

17. Goñi JR, de la Cruz X, Orozco M (2004) Triplex - forming oligonucleotide target sequences in the human genome, Nucleic Acids Research 32: 54-360

18. Goñi JR, Vaquerizas JM, Dopazo J, Orozco M (2006) Exploring the reasons for the large density of triplex-forming oligonucleotide target sequences in the human regulatory regions, BMC Genomics 7: 63

19. Aviñó A, Frieden M, Morales JC, García de la Torre B, Güimil García R, Azorín F, Gelpí JL, Orozco M, González C, Eritja R (2002) Properties of triple helices formed by parallel-stranded hairpins containing 8-aminopurines. Nucleic Acids Research 30: 2609-2619 
20. Aviñó A, Morales JC, Frieden M, de la Torre BG, Güimil García R, Cubero E, Luque FJ, Orozco M, Azorín F, Eritja R (2001) Parallel-stranded hairpins containing 8-aminopurines. Novel efficient probes for triple-helix formation, Bioorganic \& Medicinal Chemistry Letters 11:1761-1763

21. Nadal A, Eritja R, Esteve T, Pla M (2005) "Parallel" and "antiparallel tail-clamps" increase the efficiency of triplex formation with structured DNA and RNA targets, ChemBioChem, 6: 1034-1042.

22. Nadal A, Coll A, Aviñó A, Esteve T, Eritja R, Pla M (2006) Efficient sequence specific purification of Listeria innocua mRNA Species by triplex affinity capture with parallel tail clamps. ChemBioChem 7: $1039-1047$

23. Carrascosa LG, Gómez-Montes S, Aviñó A, Nadal A, Pla M, Eritja R, Lechuga LM (2012) Sensitive and label-free biosensing of RNA with predicted secondary structures by a triplex affinity capture method, Nucleic Acids Research 40: e56.

24. Takagi T, Iio A, Nakagawa Y, Naoe T, Tanigawa N, Akao Y. (2009) Decreased expression of microRNAs-143 and -145 in human gastric cancers, Oncology 77: 12-21

25. Mergny JL, Lacroix L (1998) Kinetics and thermodynamics of i-DNA formation: phosphodiester versus modified oligodeoxynucleotides. Nucleic Acids Research 26: 4797-4803

26. Gowers DM, Fox KD (1999) Towards mixed sequence recognition by triple helix formation. Nucleic Acids Research 27: 1569-1577

27. Sugimoto N, Wu P, Hara H, Kawamoto Y (2001) pH and cation effects on the properties of parallel pyrimidine motif DNA triplexes Biochemistry, 40: 9396-9405.

28. Liu K, Miles HT, Frazier J, Sasisekharan V (1993) A novel DNA duplex. A parallel-stranded DNA helix with Hoogsteen base pairing. Biochemistry, 32: 11802-11809

29. Carascosa L G, Calle A, Lechuga L M (2009) Label-free detection of DNA mutations by SPR: application to the early detection of inherited breast cancer. Anal. Bioanal. Chem. 393: 1173-1182 
30. McGuffie EM, Catapano, CV (2002) Design of a novel triple helix-forming oligodeoxyribonucleotides directed to the major promoter of the c-myc gene. Nucleic Acids Res. 30: 2701-2709

31. Winters TA (2000) Gene targeted agents: new opportunities for rational drug development. Curr. Opin. Mol.Ther. 2: 670-681

32. Toscano-Garibaya JD, Aquino-Jarquin G (2014) Transcriptional regulation mechanism mediated by miRNA-DNA-DNA triplex structure stabilized by Argonaute. Biochimica et Biophysica Acta 1839: 1079-1083

33. Kanak, M. Alseiari M, Balasubramanian P, Addanki K, Aggarwal M, Noorali S, Kalsum A, Mahalingam K, Pace G, Panasik N, Bagasra O (2010) Triplex-forming MicroRNAs Form Stable Complexes With HIV-1 Provirus and Inhibit its Replication. Appl. Immunohistochem. Mol. Morphol. 18:532-545 